\title{
Editorial: Coding and Cryptography 2019
}

\author{
Anne Canteaut ${ }^{1} \cdot$ Gohar Kyureghyan ${ }^{2} \cdot$ Alexander Pott $^{3} \cdot$ Felix Ulmer $^{4}$
}

Published online: 29 August 2020

(c) Springer Science+Business Media, LLC, part of Springer Nature 2020

This volume contains 16 refereed papers addressing a wide range of topics in coding and cryptography. The selected papers are the full journal versions of extended abstracts accepted for presentation at the International Workshop on Coding and Cryptography (WCC 2019) held in Saint-Jacut-de-la-Mer, March 31 to April 5, 2019. The aim of WCC is to bring together researchers in all aspects of coding theory, cryptography and related areas, theoretical or applied. The variety of topics covered by WCC is a recurring feature of the workshop and attracts many colleagues.

Each of the 68 extended abstracts originally submitted to the workshop were reviewed by at least two members of the Program Committee. As a result of the screening process, 39 papers were selected for the presentation at the workshop on Coding and Cryptography together with three invited talks by Alain Couvreur (Inria Saclay, France), Venkatesan Guruswami (Carnegie Mellon University, USA) and Gohar Kyureghyan (Universität Rostock, Germany). Each of the full-version submissions was once again thoroughly refereed by at least two anonymous reviewers. This volume is the end result of this second full-fledged reviewing process. We are grateful to the reviewers who contributed to guaranteeing the high standards of these papers. We would like to thank all the authors and all the other participants of the WCC 2019 for making it a highly enjoyable and stimulating event.

Publisher's Note Springer Nature remains neutral with regard to jurisdictional claims in published maps and institutional affiliations.

\footnotetext{
$凶$ Anne Canteaut

anne.canteaut@inria.fr

Gohar Kyureghyan

gohar.kyureghyan@uni-rostock.de

Alexander Pott

alexander.pott@ovgu.de

Felix Ulmer

felix.ulmer@univ-rennes1.fr

1 Inria, Paris, France

2 Universität Rostock, Rostock, Germany

3 Otto-von-Guericke-Universität, Magdeburg, Germany

4 Université de Rennes 1, Rennes, France
} 